\title{
MYOPATHY SECONDARY TO LUNG ADENOCARCINOMA: CASE REPORT
}

\section{Bruna Luiza Oliveira Lima1,*, Fernanda de Costa Ferreira Guerra ${ }^{1}$, Pedro Henrique de Souza Serafin ${ }^{1}$, João Pedro Costa dos} Santos Guerra ${ }^{1}$, Camila de Oliveira Trevisan Coutinho ${ }^{1}$

1. Universidade Federal de Mato Grosso, Sinop (MT), Brazil.

*Corresponding author: bbrunalima99@gmail.com

\section{BACKGROUND}

Paraneoplastic syndromes (PNS) are clinical manifestations that are associated with tumors. They occur in about $10 \%$ of lung cancer patients, and may precede the diagnosis or appear late in the course of the disease, with cutaneous, endocrine or metabolic, hematological, neurological and renal presentations being more common. Myopathies are primary structural and/or functional disorders that cover the muscle and can be secondary to lung adenocarcinoma, being part of the rare presentation of paraneoplastic syndromes. In elderly patients, myopathies resulting from PNS are more frequent and, therefore, there is a significant importance in including this in the diagnostic hypotheses. Myopathies are disorders confined to the muscle without structural abnormality of the peripheral nerve and are classified into groups: muscular dystrophies, congenital myopathy, myotonic dystrophy, inflammatory myopathies, metabolic myopathies and ion channel diseases. In addition to inflammatory and mechanical causes, it is important to associate myopathies with paraneoplastic syndromes.

\section{CASE REPORT}

Female patient, 64 years old, reported that a muscle weakness in the shoulder girdle started 7 months ago associated with paresthesia in the upper limbs, lack of appetite and weight loss ( $5 \mathrm{~kg} / 7$ months). Physical examination revealed muscle strength grade 4 in the upper limbs and grade 5 in the lower limbs. Sequentially, tests were requested that showed CPK: 171, LDH: 1250, AST: 47, ALT: 50, alkaline phosphatase: 258, ESR: 36, CRP: 145, normal protein electrophoresis and negative antibodies. It was also evidenced in the lung X-ray, a mass at the apex of the right lung and in the electroneuromyography of the four limbs, myotonic discharges, suggestive of myotonic myopathy. Subsequently, she was referred to oncology and diagnosed with lung adenocarcinoma showing paraneoplastic myopathy.

\section{CONCLUSION}

Although infrequent, myopathies can be diagnosed as paraneoplastic syndrome in lung adenocarcinomas. Therefore, it is necessary that the rheumatologist associate manifestations of myopathies in front of patients with lung tumors. 